\title{
Medicare Prescription Drug Plan Coverage of Hormone Therapies Used by Transgender Individuals
}

\author{
Michael T. Solotke, ${ }^{1, *}$ Patrick Liu, ${ }^{1, *}$ Sanket S. Dhruva, MD, MHS, ${ }^{2,3}$ Barbara Gulanski, MD, MPH, ${ }^{4,5}$ \\ Nilay D. Shah, PhD, ${ }^{6}$ and Joseph S. Ross, MD, MHS ${ }^{7-10}$
}

\begin{abstract}
Purpose: Although pharmacologic hormone therapy represents one of the mainstays of gender-affirming therapy for transgender individuals, there are many access barriers for these therapies, including insurance coverage of these drugs. The purpose of this study was to examine Medicare coverage of hormone therapies used by transgender individuals.

Methods: Using Centers for Medicare and Medicaid Services prescription drug plan formulary files, we determined plan coverage, coverage restrictions, and out-of-pocket (OOP) costs for all 10 drugs recommended in the 2009 and 2017 Endocrine Society treatment guidelines for transgender patients.

Results: For masculinizing therapies, the proportion of plans providing unrestricted coverage ranged from $22 \%$ to $79 \%$ in 2010 and from 5\% to $75 \%$ in 2018 . For feminizing therapies, the proportion providing unrestricted coverage ranged from 24\% to $100 \%$ in 2010 and from $13 \%$ to $100 \%$ in 2018. Median annual OOP costs for masculinizing therapies ranged from $\$ 232$ to $\$ 1112$ in 2010 and from $\$ 180$ to $\$ 2176$ in 2018. For feminizing therapies, OOP costs ranged from $\$ 84$ to $\$ 2716$ in 2010 and from $\$ 72$ to $\$ 3792$ in 2018.

Conclusion: Our findings highlight the variability in access to guideline-recommended hormone therapies for individuals insured through Medicare.
\end{abstract}

Keywords: Endocrine Society guideline, gender-affirming therapy, hormone therapy, Medicare prescription drug plans, transgender

\section{Introduction}

$\mathbf{T}$ The Institute of Medicine (now called the National Academy of Medicine) identified the transgender adult population as an at-risk group because these individuals face both an increased disease burden and reduced access to health care services. ${ }^{1}$ Many transgender individuals are uninsured, do not have a health care provider, or are unable to afford a health care appointment when they need it. ${ }^{2}$ Transgender people also commonly experience discrimination in health care settings, and nearly one quarter of trans- gender patients report needing to teach their clinician about transgender health care. ${ }^{3}$ Even after securing access to an appropriately trained clinician, gaining access to guidelinerecommended therapies can be challenging for transgender individuals.

The mainstays of treatment for transgender individuals include access to mental health care, pharmacologic therapy to suppress endogenous hormones and/or hormone therapy (HT) to maintain sex hormones within the physiologic range for the patient's affirmed gender, and, for some individuals, gender-affirming surgery. HT is a mainstay of

\footnotetext{
${ }^{1}$ Yale School of Medicine, Yale University, New Haven, Connecticut.

${ }^{2}$ Section of Cardiology, Department of Medicine, San Francisco Veterans Affairs Medical Center, San Francisco, California.

${ }^{3}$ UCSF School of Medicine, University of California, San Francisco, San Francisco, California.

${ }^{4}$ Section of Endocrinology, Veterans Affairs Connecticut Healthcare System, West Haven, Connecticut.

${ }^{5}$ Section of Endocrinology, Department of Internal Medicine, Yale School of Medicine, Yale University, New Haven, Connecticut.

${ }^{6}$ Division of Health Care Policy and Research and Kern Center for the Science of Health Care Delivery, Mayo Clinic, Rochester, Minnesota.

${ }^{7}$ National Clinician Scholars Program, and ${ }^{8}$ Section of General Internal Medicine, Department of Internal Medicine, Yale School of Medicine, Yale University, New Haven, Connecticut.

${ }^{9}$ Department of Health Policy and Management, Yale School of Public Health, Yale University, New Haven, Connecticut.

${ }^{10}$ Center for Outcomes Research and Evaluation, Yale-New Haven Hospital, New Haven, Connecticut.

*Co-first authors.
} 
gender-affirming therapy because it can induce physical changes consistent with one's affirmed gender identity, because it is safe when managed by a trained clinician, because it is associated with an improvement in mental well-being, and because it is commonly required before undergoing gender-affirming surgery. ${ }^{4-7}$ Although gender-affirming surgery is clinically indicated for transgender individuals who desire it and meet certain eligibility requirements, HT is more commonly used in practice because many transgender people do not meet the above criteria or are unable to afford surgery. ${ }^{6}$ The Endocrine Society and the World Professional Association for Transgender Health (WPATH) have published evidence-based clinical guidelines on medical treatment and care for transgender individuals. ${ }^{6,7}$ To underscore the medical necessity of these treatments, the Endocrine Society recently issued a position statement emphasizing the need for payers to cover prescribed medical interventions for transgender patients. ${ }^{8}$

However, despite the existence of established clinical guidelines and the medical necessity of these treatments, one U.S. survey of transgender individuals found that $25 \%$ reported being denied insurance coverage for hormone therapies. ${ }^{3}$ There has been no systematic examination of formulary coverage for hormone therapies and it is unknown whether and to what degree health plans for Medicare beneficiaries are providing coverage for these therapies for older transgender adults, a particularly vulnerable subgroup who may face several unique challenges, such as increased identity concealment in health care settings and difficulty identifying clinicians who can manage HT in the setting of medical comorbidities. ${ }^{9-12}$ Accordingly, we examined Medicare prescription drug plan (PDP) formulary coverage and restrictions for those hormone therapies recommended in the 2009 and 2017 Endocrine Society treatment guidelines, ${ }^{6,13}$ including out-of-pocket (OOP) costs associated with hormone therapies. We expect our findings to help provide a better understanding of the cost and access barriers faced by the transgender population.

\section{Methods \\ Data sources}

We used Centers for Medicare and Medicaid Services (CMS) PDP formulary files from the second quarters of 2010, 2014, and 2018. These files provide details about plan structure and coverage for all Medicare Advantage and stand-alone plans, but do not provide information about the Medicare beneficiaries enrolled in each plan. Medicare Advantage PDPs (MA-PDPs) provide drug coverage in addition to benefits for other medical services, whereas standalone PDPs solely provide prescription medication coverage. We limited our study to plans that submitted full details about cost-sharing, coverage phase, and tier structures for the drugs in our sample. We excluded special needs plans, which are Medicare advantage plans that restrict enrollment to institutionalized individuals, dual eligibility, and individuals with severe or disabling chronic conditions. We selected the years 2010 and 2018 to allow time for plans to incorporate clinical recommendations from the 2009 and 2017 Endocrine Society guidelines; we selected 2014 because it was the midpoint between 2010 and $2018 .{ }^{6,13}$ As this project used publicly available data that did not involve human subjects, it was exempt from review by the Yale Human Research Protection Program.

\section{Drug sample}

We examined all drugs recommended in the 2009 and 2017 Endocrine Society treatment guidelines for transgender patients, ${ }^{6,13}$ which differed in recommended dosage but were otherwise the same, and included all available generic and brand-name formulations that were available during the entire duration of the study. HT for trans-masculine individuals typically consists of testosterone in patch, gel, or injection form. Trans-feminine individuals may take estrogen monotherapy (available as a pill, patch, injection, or gel) or a multidrug regimen consisting of estrogen and spironolactone. Leuprolide may also be included in multidrug regimens for trans-feminine people. ${ }^{6}$ For medications of the same form of delivery that had multiple generic and brand name formulations available (e.g., multiple versions of oral estradiol), we reviewed all formulations identified in the formulary files, but limited our study to the formulation that had the greatest number of plans offering the drug with unrestricted coverage, as defined below. We report results using the generic or brand name, depending on which formulation was included in our final drug sample.

\section{Outcome measures: plan coverage and coverage restrictions}

To examine access to HT, we first determined plan coverage and coverage restrictions for each drug in our sample. Every Medicare PDP has a formulary, which is a list that assigns tiers and coverage restrictions through utilization management strategies for covered medications. Coverage restrictions include prior authorization, step therapy, and quantity limits. ${ }^{14}$ Prior authorization requires prescribers to receive approval before prescribing a drug and is intended to ensure that patients meet certain clinical criteria to warrant a particular therapy. ${ }^{15}$ Step therapy requires the use of another drug before a particular drug can be approved for reimbursement. Drugs excluded from a formulary are typically not reimbursed by the payer, and beneficiaries are responsible for the full cost of these drugs.

\section{Outcome measures: beneficiary OOP costs}

We also determined required beneficiary OOP costs for each drug of interest, adjusted for inflation using June 2018 as the index month and year. ${ }^{16}$ Because the frequency of administration varies from daily (for patches and pills) to weekly, biweekly, or monthly (for injections), we used cost information for a 30-day supply of each drug in our sample. In addition, because dose ranges can be highly variable and oral medications of lower dosages can be combined to achieve a higher dose, we used the lowest dose of the dose ranges listed in the 2017 Endocrine Society guideline to calculate costs. ${ }^{6}$ We then chose the least expensive dosequantity combination to reach the lowest dose of the listed dose range. This approach allowed us to provide the most conservative estimate of OOP costs required of transgender beneficiaries. 
Medicare PDPs must set their deductible phase, initial coverage zone, coverage gap, and catastrophic coverage zone within parameters set annually by CMS, but they have flexibility within these limits. ${ }^{17}$ For example, some plans may require beneficiaries to enter a deductible phase where beneficiaries typically pay the full cost of the drug before moving into the initial coverage zone. Other plans have a $\$ 0$ deductible, thereby immediately sharing in the medication costs of their enrolled beneficiaries. We used each plan's specific coverage structure, rather than the standard Part D benefit, when calculating our main outcomes.

\section{Statistical analyses}

For each drug, descriptive statistics were used to determine the percentage of Medicare PDPs providing coverage without any prior authorization requirement, step therapy requirement, or quantity limit (unrestricted coverage); the percentage providing coverage with one or more restrictions (restricted coverage); and the percentage not providing coverage. For each drug, we then used descriptive statistics to calculate median annual OOP beneficiary copayment (using the 30-day supply cost) among all plans offering coverage. We examined annual costs because these medications are typically used on a long-term basis. Results were stratified by feminizing versus masculinizing therapies and by plan type (stand-alone PDP vs. MA-PDP) for each of the years (i.e., 2010, 2014, and 2018) because prior analyses have shown differences in OOP costs between the two plan types. ${ }^{18}$ We used the $\chi^{2}$ test to compare the proportion of plans providing coverage and the Mann-Whitney $U$ test to compare median OOP costs in 2010 and 2018, overall and stratified by plan type. We used the Bonferroni correction to adjust for multiple comparisons, using a $p$ value of 0.025 as the threshold for statistical significance. All statistical analyses were performed using R Studio version 1.1.423 (RStudio, Inc., Boston, MA).

\section{Results}

There were 2978 Medicare PDPs in 2010, 2857 in 2014, and 3052 in 2018 (Table 1). The proportion of MA-PDPs relative to stand-alone PDPs grew over time, from 54.5\% $(n=1622)$ MA-PDPs versus $45.5 \%(n=1356)$ stand-alone PDPs in 2010 to $74.4 \%(n=2272)$ MA-PDPs versus $25.6 \%$ $(n=780)$ stand-alone PDPs in 2018.

\section{Drug sample}

There were 10 drugs recommended in the 2009 and 2017 Endocrine Society treatment guidelines for transgender

Table 1. Number of Medicare Advantage Prescription Drug Plans and Stand-Alone Prescription Drug Plans in 2010, 2014, and 2018

\begin{tabular}{lccr}
\hline & 2010 & 2014 & 2018 \\
\hline Total number of & 2978 & 2857 & 3052 \\
$\quad$ PDPs in sample & & & \\
MA-PDPs, $n(\%)$ & $1622(54.5)$ & $1758(61.5)$ & $2272(74.4)$ \\
Stand-alone & $1356(45.5)$ & $1099(38.5)$ & $780(25.6)$ \\
$\quad$ PDPs, $n(\%)$ & & & \\
\hline
\end{tabular}

MA, Medicare advantage; PDP, prescription drug plan.
Table 2. Hormone Therapy Drug Sample and Dosages Analyzed

\begin{tabular}{ll}
\hline Drug name & Dose analyzed \\
\hline $\begin{array}{l}\text { Masculinizing therapies } \\
\text { Gel (Testim) }\end{array}$ & $50 \mathrm{mg}$ gel \\
packet daily \\
Transdermal patch (Androderm) & $4 \mathrm{mg} \mathrm{daily}$ \\
Parenteral (testosterone-cypionate) & $50 \mathrm{mg}$ weekly \\
Parenteral (testosterone-enanthate) & $50 \mathrm{mg}$ weekly \\
Feminizing therapies & $2 \mathrm{mg}$ daily \\
Oral (estradiol) & $0.1 \mathrm{mg}$ daily \\
Transdermal patch (estradiol) & $20 \mathrm{mg}$ every \\
Parenteral (estradiol-valerate) & other week \\
Parenteral (Depo-Estradiol) & $5 \mathrm{mg}$ weekly \\
Oral (spironolactone) & $100 \mathrm{mg}$ daily \\
Parenteral (Lupron-Depot) & $3.75 \mathrm{mg}$ monthly \\
\hline
\end{tabular}

patients that met our study inclusion criteria: four masculinizing agents (all testosterone agents) and six feminizing agents (including four forms of estrogen derivatives, one antiandrogen agent, and one gonadotropin-releasing hormone [GnRH] agonist) (Table 2). ${ }^{6,13}$

\section{Plan coverage}

The percentage of plans providing unrestricted, restricted, and no coverage for medications was significantly different between 2010 and $2018(p<0.005)$. Overall, the proportion of plans providing no coverage or restricted coverage generally increased or stayed the same between 2010 and 2018, whereas the proportion providing unrestricted coverage decreased (Figs. 1 and 2). In addition, the percentage of plans providing unrestricted, restricted, and no coverage was significantly different between MA-PDPs and stand-alone PDPs in $2018(p<0.005)$ but not in $2010(p=0.28)$.

For masculinizing therapies, the proportion of plans providing unrestricted coverage ranged from 22\% (Androderm) to $79 \%$ (testosterone-cypionate and -enanthate) in 2010 and from 5\% (Testim) to 75\% (testosterone-cypionate) in 2018 (Fig. 1). Notably, for Testim, the proportion of plans providing unrestricted coverage was $29 \%$ in $2010,13 \%$ in 2014, and 5\% in 2018; and for Androderm, the proportion providing unrestricted coverage was $22 \%$ in $2010,22 \%$ in 2014 , and $8 \%$ in 2018 . The proportion of plans providing restricted coverage varied from $19 \%$ (testosterone-cypionate) to $66 \%$ (Androderm) in 2010 and from 17\% (Testim) to $54 \%$ (Androderm) in 2018. Finally, the proportion of plans offering no coverage ranged from $1 \%$ (testosterone-enanthate) to 28\% (Testim) in 2010 and from 0\% (testosterone-cypionate) to 78\% (Testim) in 2018. In 2010 and 2018, for two of four therapies, plans more often provided restricted as opposed to unrestricted coverage. These results stratified by MA-PDPs and stand-alone PDPs are provided in Table 3.

For feminizing therapies, the proportion of plans providing unrestricted coverage ranged from 24\% (LupronDepot) to $100 \%$ (oral estradiol and spironolactone) in 2010 and from 13\% (transdermal estradiol) to 100\% (spironolactone) in 2018 (Fig. 2). Notably, for transdermal estradiol, the proportion of plans providing unrestricted coverage was 


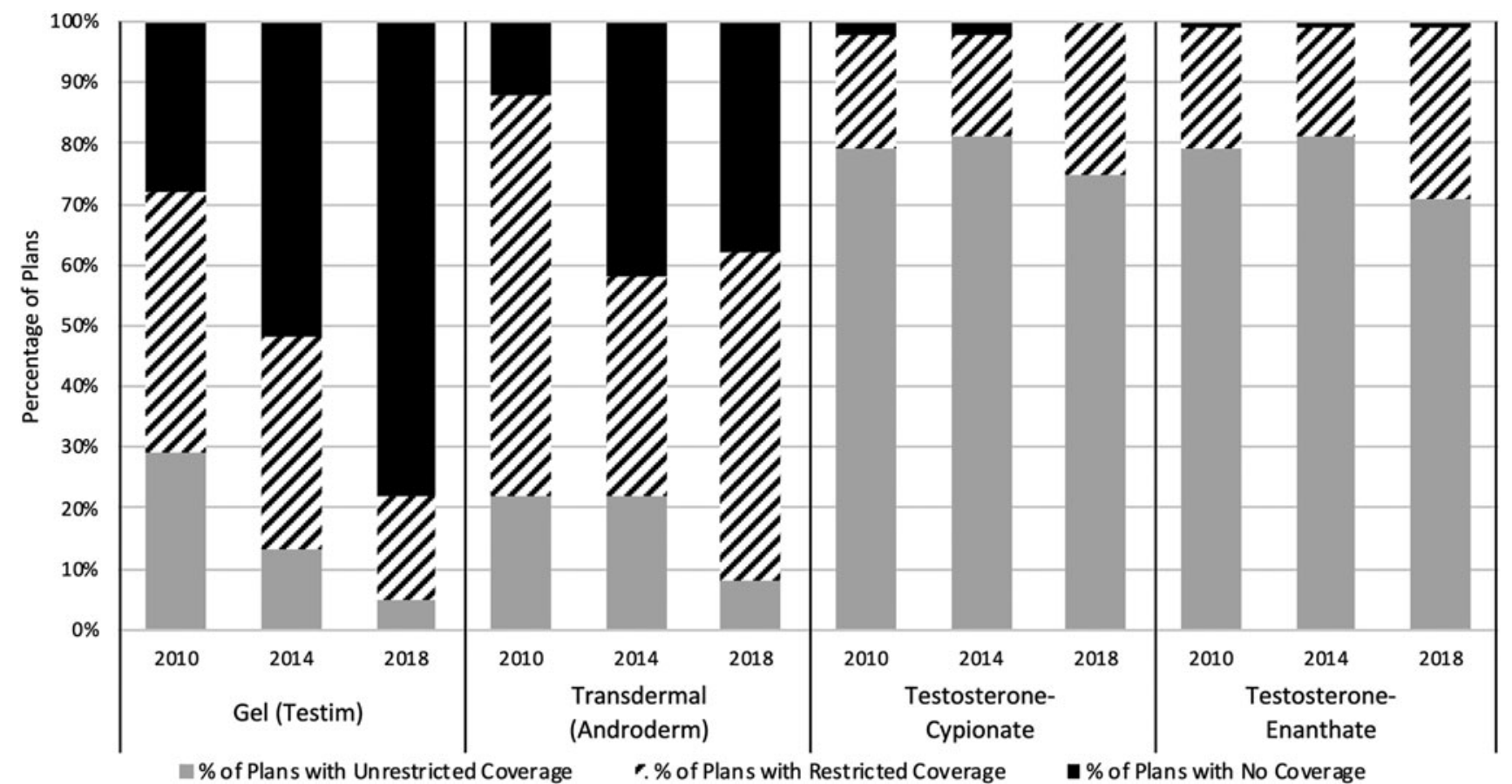

FIG. 1. Medicare PDP coverage and restrictions for masculinizing hormone therapies in 2010, 2014, and 2018. PDP, prescription drug plan.

$49 \%$ in $2010,19 \%$ in 2014 , and $13 \%$ in 2018 . Across all plans, the proportion of plans providing restricted coverage for feminizing therapies varied from $0 \%$ (oral estradiol, estradiol-valerate, and spironolactone) to $71 \%$ (LupronDepot) in 2010 and from $0 \%$ (estradiol-valerate and spironolactone) to $80 \%$ (Lupron-Depot) in 2018. The proportion of plans providing no coverage ranged from $0 \%$ (oral estradiol and spironolactone) to $39 \%$ (Depo-Estradiol) in 2010 and from $0 \%$ (oral estradiol, spironolactone, and LupronDepot) to $40 \%$ (Depo-Estradiol) in 2018. Among plans covering the medications, one of six feminizing therapies had more plans providing restricted as opposed to unrestricted coverage in 2010, whereas this was true for three of six medications in 2018. Results by plan type are given in Table 3.

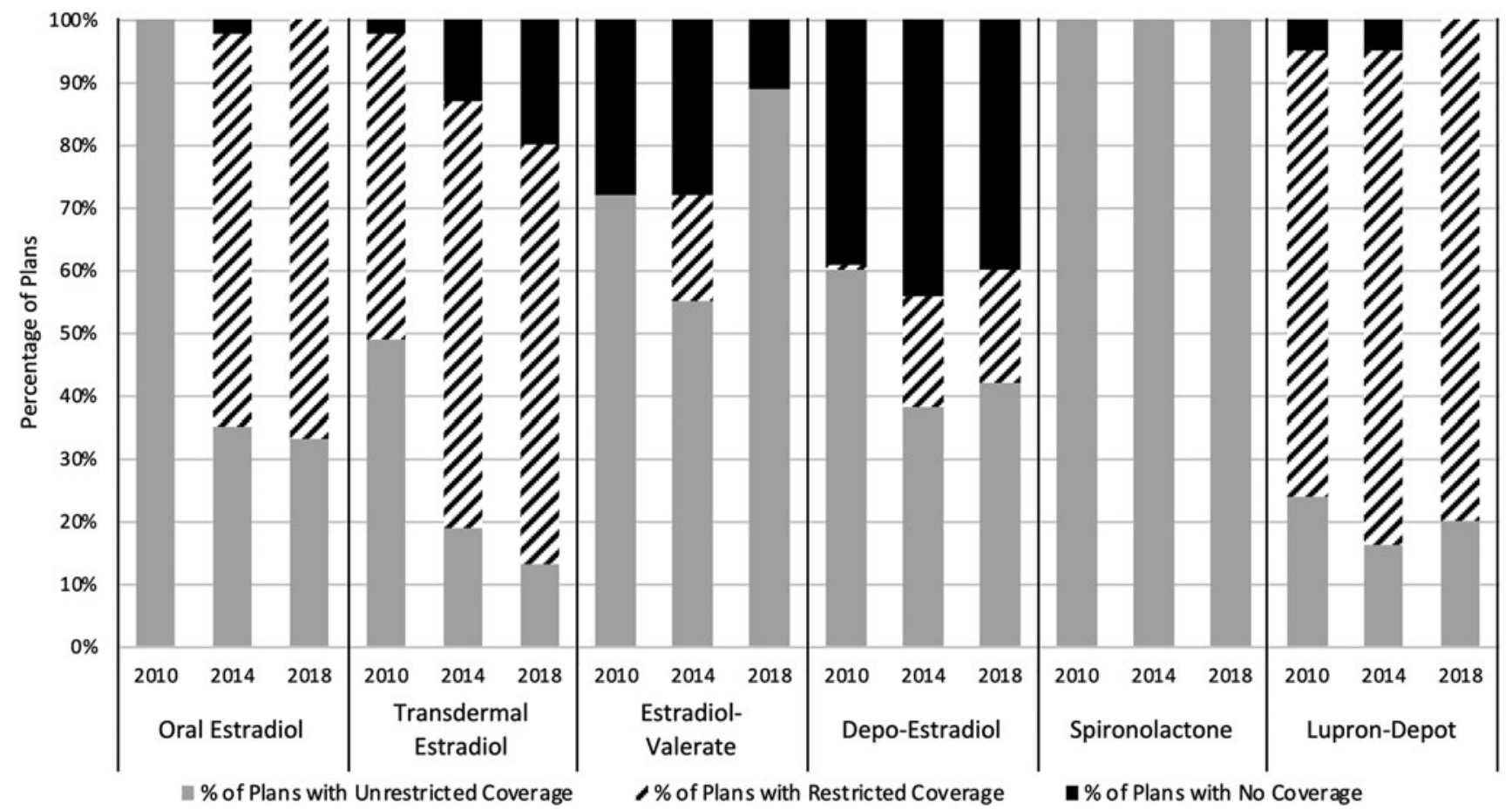

FIG. 2. Medicare PDP coverage and restrictions for feminizing hormone therapies in 2010, 2014, and 2018. 


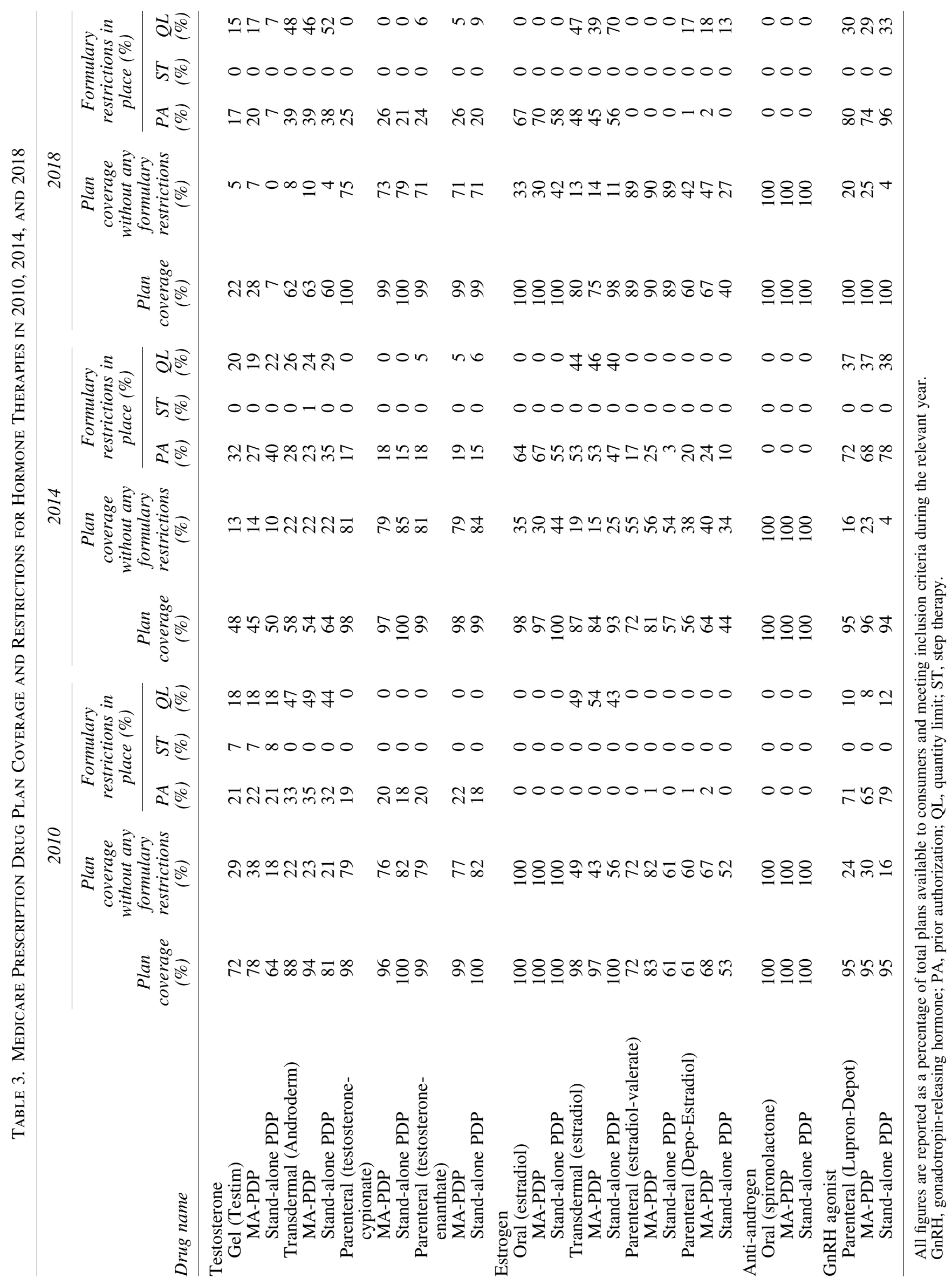




\section{OOP costs}

For masculinizing therapies, median annual OOP beneficiary costs ranged from $\$ 232$ (interquartile range [IQR], \$84-\$437, testosterone-cypionate) to \$1112 (IQR, \$788\$1205, Testim) in 2010 and from \$180 (IQR, \$114-\$253, testosterone-cypionate) to $\$ 2176$ (IQR, \$2103-\$2439, Testim) in 2018 (Fig. 3). In 2010, three of four masculinizing therapies had lower OOP costs when covered by MA-PDPs than by stand-alone PDPs; in 2018, it was two of four (Table 4).

For feminizing therapies, median annual OOP beneficiary costs ranged from $\$ 84$ (IQR, \$70-\$97, oral estradiol) to \$2716 (IQR, \$2627-\$3338, Lupron-Depot) in 2010 and from $\$ 72$ (IQR, \$12-\$144, spironolactone) to \$3792 (IQR, \$3715-\$3918, Lupron-Depot) in 2018 (Fig. 3). By plan type, in 2010, five of six feminizing therapies had lower OOP costs when covered by MA-PDPs than by stand-alone PDPs; in 2018, it was one of six (Table 4). Between 2010 and 2018, costs were significantly different for stand-alone PDPs $(p<0.005)$, but not for MA-PDPs $(p=0.027)$ (Table 4$)$.

\section{Discussion}

In this study of all drugs recommended by the 2009 and 2017 Endocrine Society guidelines for treatment of transgender people, ${ }^{6,13}$ we found substantial variability in Medicare PDP coverage and beneficiary OOP costs associated with medically necessary HT used by transgender individuals. Although several feminizing and masculinizing therapies were generally covered and associated with annual OOP costs under $\$ 500$, restricted or no coverage of the therapies was common. Medicare Advantage plans more commonly offered unrestricted coverage than their stand-alone counterparts for both feminizing and masculinizing therapies. However, while most ( 8 of 10) therapies were associated with lower OOP costs through MA-PDPs than stand-alone PDPs in 2010, MA-PDPs had higher OOP costs than their standalone counterparts (for 7 of 10 therapies) in 2018 .

Our formulary coverage analysis showed high rates of formulary restrictions, which are consistent with studies showing high and increasing rates of prior authorization and step therapy required in other drug classes, such as those used to treat rheumatoid arthritis, hepatitis $\mathrm{C}$, hyperlipidemia, and multiple sclerosis. ${ }^{19-22}$ Although formulary restrictions are typically implemented to manage spending and drug utilization, ${ }^{14}$ they can negatively impact medication fills and adherence. $^{23,24}$ Our results may provide context to the findings from a U.S. survey of transgender individuals, in which $25 \%$ of respondents reported being denied insurance coverage for hormone therapies. ${ }^{3}$ These access barriers may result in higher OOP costs and lack of adherence to HT. Although we found that a minority of HT medications are commonly provided with unrestricted coverage, this finding may reflect that these medications are most commonly used for other indications and are available in low-cost generic form.

Our OOP cost analysis showed that annual costs for HT can vary widely and are highly dependent on the medication regimen. Given the high rates of poverty and low household income in the transgender population, ${ }^{3,25}$ these costs may be prohibitive for many people. Even for individuals who can afford HT OOP costs, these expenses should be considered

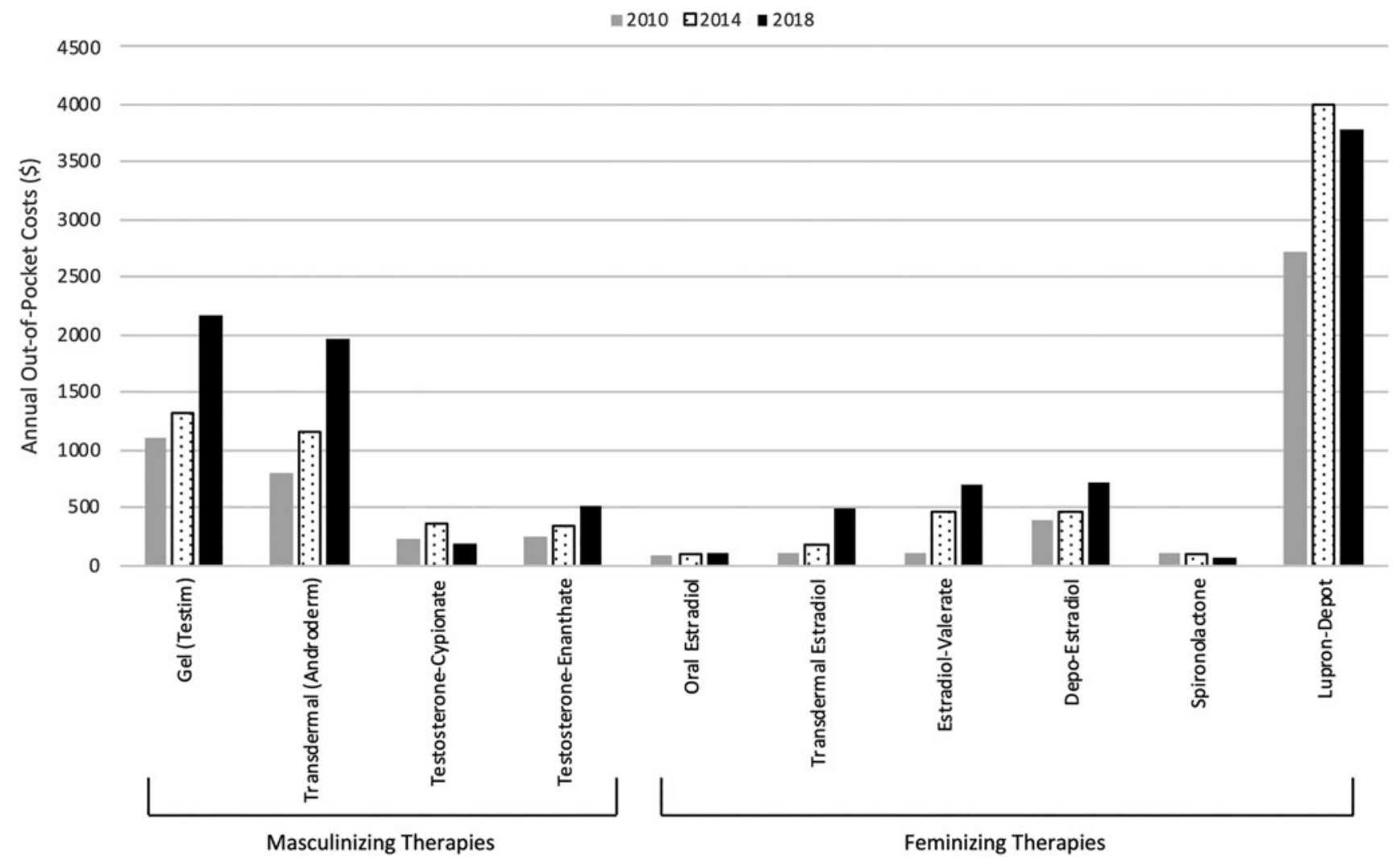

FIG. 3. Median annual required Medicare beneficiary out-of-pocket costs for hormone therapies in 2010, 2014 , and 2018. 


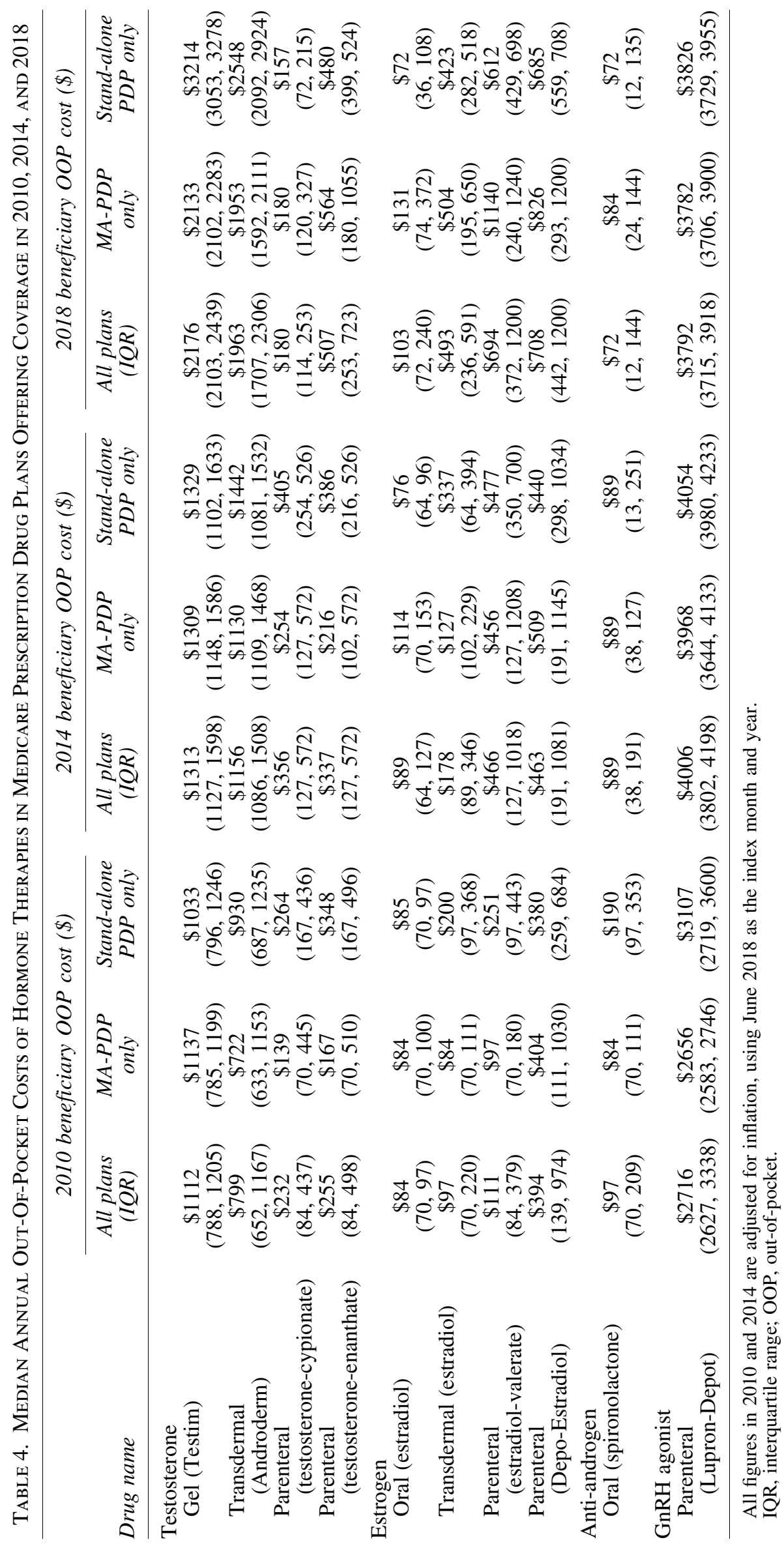


in the context of an individual's overall medication regimen. Transgender Medicare beneficiaries more commonly have multiple chronic medical conditions than cisgender beneficiaries, ${ }^{10}$ and they have more emergency department visits and hospitalizations. ${ }^{26}$ These cost and access burdens may explain why nearly 1 in 10 transgender people has acquired HT through nonlicensed sources such as friends or online. ${ }^{3}$ Our finding that OOP costs remain substantial adds to other access barriers that have been described for this population, including: delayed referrals, long waits before initial visits, shortage of clinicians able and willing to provide gender-affirming care, high rates of discrimination from clinicians, difficulty gaining and understanding insurance coverage, problems with diagnostic coding (e.g., denied reimbursements based on gender markers), and financial barriers to care. ${ }^{27-30}$

\section{Limitations}

Our study has several limitations. First, although our analysis examined for the presence of utilization management restrictions such as prior authorization, our data do not provide information about the specific details of these restrictions and therefore cannot reveal differences in access to HT for gender minority versus non gender minority beneficiaries. Second, because we did not know the number of beneficiaries enrolled in each plan, we could not weigh our results by the number of beneficiaries. Third, because there are no publicly available data about the most commonly used HT regimens and dosages in older transgender adults, our calculations may not be representative of the most commonly used medications and dosages. We selected the lower limits of the ranges provided in the 2017 Endocrine Society guideline to provide the most conservative estimate of coverage and OOP costs. ${ }^{6}$ Finally, because our data were not drawn from insurance claims or patient records, we do not know the actual OOP costs paid by beneficiaries nor do our data reflect costs paid by dual-eligible beneficiaries or low-income subsidy enrollees.

\section{Conclusion}

We found substantial variability in Medicare formulary coverage and OOP costs for HT. This is the first published analysis describing formulary coverage and OOP costs for HT used by transgender individuals, and it highlights the substantial access and cost barriers faced by this marginalized population. Further research is needed to understand overall medication costs faced by transgender individuals and the impact of this cost burden on their health and quality of life.

\section{Disclaimer}

Preliminary analyses of this research were presented at the LGBT Health Workforce Conference in New York, NY, on May 3-4, 2019.

\section{Author Disclosure Statement}

In the past 3 years, Dr. J.S.R. has received research support through Yale University from Johnson and Johnson to develop methods of clinical trial data sharing, from Medtronic, Inc., and the Food and Drug Administration to develop methods for postmarket surveillance of medical devices
(U01FD004585), from the Food and Drug Administration to establish the Yale-Mayo Clinic Center for Excellence in Regulatory Science and Innovation program (U01FD005938), from the Blue Cross Blue Shield Association to better understand medical technology evaluation, from the Centers for Medicare and Medicaid Services to develop and maintain performance measures that are used for public reporting (HHSM-500-2013-13018I), from the Agency for Healthcare Research and Quality (R01HS022882), from the National Heart, Lung, and Blood Institute of the National Institutes of Health (R01HS025164), and from the Laura and John Arnold Foundation to establish the Good Pharma Scorecard at Bioethics International and the Collaboration on Research Integrity and Transparency at Yale. In the past 3 years, Dr. N.D.S. has received research support through Mayo Clinic from the Food and Drug Administration to establish the Yale-Mayo Clinic Center for Excellence in Regulatory Science and Innovation program (U01FD005938), from the Centers for Medicare and Medicaid Innovation under the Transforming Clinical Practice Initiative, from the Agency for Healthcare Research and Quality (U19HS024075, R01HS025164, R01HS025402, R03HS025517), from the National Heart, Lung, and Blood Institute of the National Institutes of Health (R56HL130496, R01HL131535), from the National Science Foundation, and from the PatientCentered Outcomes Research Institute to develop a Clinical Data Research Network. All other authors have no competing interests to disclose.

\section{Funding Information}

No funding was received for this article.

\section{References}

1. Institute of Medicine (US) Committee on Lesbian, Gay, Bisexual, and Transgender Health Issues and Research Gaps and Opportunities: The Health of Lesbian, Gay, Bisexual, and Transgender People: Building a Foundation for Better Understanding. Washington, DC: National Academies Press, 2011.

2. Meyer IH, Brown TN, Herman JL, et al.: Demographic characteristics and health status of transgender adults in select US regions: Behavioral Risk Factor Surveillance System, 2014. Am J Public Health 2017;107:582-589.

3. James SE, Herman JL, Rankin S, et al.: The Report of the 2015 U.S. Transgender Survey. Washington, DC: National Center for Transgender Equality, 2016.

4. Weinand JD, Safer JD: Hormone therapy in transgender adults is safe with provider supervision; A review of hormone therapy sequelae for transgender individuals. J Clin Transl Endocrinol 2015;2:55-60.

5. Costa R, Colizzi M: The effect of cross-sex hormonal treatment on gender dysphoria individuals' mental health: A systematic review. Neuropsychiatr Dis Treat 2016;12: 1953-1966.

6. Hembree WC, Cohen-Kettenis PT, Gooren L, et al.: Endocrine treatment of gender-dysphoric/gender-incongruent persons: An Endocrine Society clinical practice guideline. J Clin Endocrinol Metab 2017;102:3869-3903.

7. Coleman E, Bockting W, Botzer M, et al.: Standards of care for the health of transsexual, transgender, and gendernonconforming people, version 7. Int J Transgend 2012; 13:165-232. 
8. Endocrine Society: Transgender Health: An Endocrine Society Position Statement. 2017. Available at www.endo crine.org/advocacy/priorities-and-positions/transgender-health Accessed February 26, 2020.

9. Proctor K, Haffer SC, Ewald E, et al.: Identifying the transgender population in the Medicare program. Transgend Health 2016;1:250-265.

10. Dragon CN, Guerino P, Ewald E, Laffan AM: Transgender Medicare beneficiaries and chronic conditions: Exploring fee-for-service claims data. LGBT Health 2017;4:404411.

11. Ettner R: Care of the elderly transgender patient. Curr Opin Endocrinol Diabetes Obes 2013;20:580-584.

12. Johnson K, Yarns BC, Abrams JM, et al.: Gay and gray session: An interdisciplinary approach to transgender aging. Am J Geriatr Psychiatry 2018;26:719-738.

13. Hembree WC, Cohen-Kettenis P, Delemarre-van de Waal HA, et al.: Endocrine treatment of transsexual persons: An Endocrine Society clinical practice guideline. J Clin Endocrinol Metab 2009;94:3132-3154.

14. Academy of Managed Care Pharmacy: Formulary Management. 2019. Available at https://www.amcp.org/about/ managed-care-pharmacy-101/concepts-managed-care-phar macy/formulary-management Accessed February 26, 2020.

15. Academy of Managed Care Pharmacy: Concepts in Managed Care Pharmacy: Prior Authorization. 2019. Available at https://www.amcp.org/about/managed-care-pharmacy101/concepts-managed-care-pharmacy/prior-authorization Accessed February 26, 2020.

16. U.S. Bureau of Labor Statistics: CPI Inflation Calculator. Available at https://www.bls.gov/data/inflation_calculator .htm Accessed February 26, 2020.

17. Medicare Interactive: Phases of Part D coverage. 2020. Available at https://www.medicareinteractive.org/get-answers/ medicare-prescription-drug-coverage-part-d/medicare-partd-costs/phases-of-part-d-coverage Accessed February 26, 2020 .

18. Liu P, Dhruva SS, Shah ND, Ross JS: Medicare beneficiary out-of-pocket costs for generic cardiovascular medications available through $\$ 4$ generic drug discount programs. Ann Intern Med 2018;169:817-819.

19. Yazdany J, Dudley RA, Chen R, et al.: Coverage for highcost specialty drugs for rheumatoid arthritis in Medicare Part D. Arthritis Rheumatol 2015;67:1474-1480.

20. Jung JK, Feldman R, Cheong C, et al.: Coverage for hepatitis C drugs in Medicare Part D. Am J Manag Care 2016; 22:Sp220-Sp226.
21. Kazi DS, Lu CY, Lin GA, et al.: Nationwide coverage and cost-sharing for PCSK9 inhibitors among Medicare Part D plans. JAMA Cardiol 2017;2:1164-1166.

22. Hartung DM, Johnston KA, Irwin A, et al.: Trends in coverage for disease-modifying therapies for multiple sclerosis in Medicare Part D. Health Aff (Millwood) 2019;38:303-312.

23. Happe LE, Clark D, Holliday E, Young T: A systematic literature review assessing the directional impact of managed care formulary restrictions on medication adherence, clinical outcomes, economic outcomes, and health care resource utilization. J Manag Care Spec Pharm 2014;20:677-684.

24. Park Y, Raza S, George A, et al.: The effect of formulary restrictions on patient and payer outcomes: A systematic literature review. J Manag Care Spec Pharm 2017;23:893-901.

25. Baker KE: Findings from the Behavioral Risk Factor Surveillance System on health-related quality of life among US transgender adults, 2014-2017. JAMA Intern Med 2019;179:1141-1144.

26. Progovac AM, Cook BL, Mullin BO, et al.: Identifying gender minority patients' health and health care needs in administrative claims data. Health Aff (Millwood) 2018; 37:413-420.

27. Jaffee KD, Shires DA, Stroumsa D: Discrimination and delayed health care among transgender women and men: Implications for improving medical education and health care delivery. Med Care 2016;54:1010-1016.

28. Grant JM, Mottet LA, Tanis J, et al.: Injustice at Every Turn: A Report of the National Transgender Discrimination Survey. Washington, DC: National Center for Transgender Equality and National Gay and Lesbian Task Force, 2011.

29. Dowd S: Transgender health equity: Patients say providers lack expertise and understanding of transgender health needs. Health Affairs, 2017. Available at https://www .healthaffairs.org/do/10.1377/hblog20170329.059403/full/ Accessed February 26, 2020.

30. Safer JD: Transgender patients and health care providers. Health Aff (Millwood) 2017;36:2213.

Address correspondence to: Michael T. Solotke

Yale School of Medicine Yale University 333 Cedar Street New Haven, CT 06510

E-mail: michael.solotke@yale.edu 